\title{
SYNTHESIS AND SPECTRAL CHARACTERIZATION OF SOME NEW SUBSTITUTED BIS-SPIROCYCLOHEXANONES DERIVED FROM ACETONE
}

\author{
Mohammed S. Hussein ${ }^{\mathrm{a} *}$, Abdul Wahab J.Al-Hamdany ${ }^{\mathrm{b}}$ and Rana A.Saeed ${ }^{\mathrm{c}}$ \\ ${ }^{a}$ Dept. of Chemistry, Faculty of Science, University of Zakho, Kurdistan Ridional Government -Iraq- \\ (mohammed.huseein@uoz.edu.krd) \\ ${ }^{b}$ Dept. of Chemistry, College of Science, University of Mosul, Mosul, Iraq - (ajalhamadany@yahoo.com) \\ 'Dept. of Pharmacy,Technical, Institute Northern Technical, University Mosul, Iraq - (rana.al alaf@yahoo.com)
}

Received: Jul. 2018 / Accepted: Nov., 2018 / Published: Dec., 2018

https://doi.org/10.25271/siuoz.2018.6.4.548

\begin{abstract}
:
Diarylidene acetones (DAA) (1-5) had been prepared by the condensation of acetone with substituted benzaldehydes via ClaisenSchmidt reaction, DAA's brought to condense with anthrone to afford the title compounds (6-10) through Michael addition. The structures of the products were suggested in the light of spectral data (UV, IR, 1H\&13C-NMR).
\end{abstract}

KEYWORDS: Anthrone, spirocyclohexanones, Michael addition.

\section{INTRODUCTION}

Baeyer in 1900 was first used the name (Spirocyclane) (Rios, 2012). The quaternary carbon center and the presence of two fused rings cause a complexity of these ring structures. Natural Compounds that isolated from different plant sources are found to contain many Spiro cyclic structures (Smith and Baxendale, 2015). Spiro compounds have generated considerable interest in recent years due to their pharmacological activities (Ghandi et al, 2009; Raj and Raghnathan, 2003). Many Spiro compounds have been found to show anticancer, narcotic, anti-inflammatory and analgesic properties (Dandia et al, 2006; Sebahar and Williams, 2000; Ma and Hecht, 2004; Kang et al, 2002; Ding et al, 2005). Spiro compounds can be prepared by various methods (Jayashankaran et al, 2005; Khan et al, 2003; Marti and Carreira, 2005; Pearson, 2002; Ungureanu et al, 2001). But the reaction of dibenzalacetone with a compound having active methylene group yielding double Michael adduct would be an interesting subject of investigation. Aggarwal and co-worker carried out the reaction of dibenzylidene acetone and N,N-dimethyl barbituric acid in ethylene glycol (Aggarwal and Vij, 2014).<smiles>CCCC(=O)N1C(=O)CC(=O)N(C)C1=O</smiles><smiles>CC1C(=O)C(=O)C2(C(=O)C(C)C1=O)C(c1ccccc1)CC(=O)CC2c1ccccc1</smiles>

The reaction of dibenzylidene acetone and N,N-sub. barbituric acid.

Treatment of barbituric acid with dibenzylidene acetone in presence of ammonium acetate as a basic medium afforded a mixture of products (Assy et al, 2015).<smiles>O=C1CC(=O)[NH2+]C(=O)N1</smiles><smiles>NC(=O)CC1CC(=O)CC(c2ccccc2)C(c2ccccc2)(C2(c3ccccc3)C(=O)NC(=O)NC2=O)C1</smiles>

The reaction of dibenzylidene acetone and barbituric acid. Spiro[cyclohexanone-oxindoles] have been synthesized and evaluated in vitro for their antiproliferative effects against the protozoan Leishmania infantum. Interestingly, they appear able to kill L. infantum promastigotes and amastigotes, without significant cytotoxic effects (Scala et al, 2014).

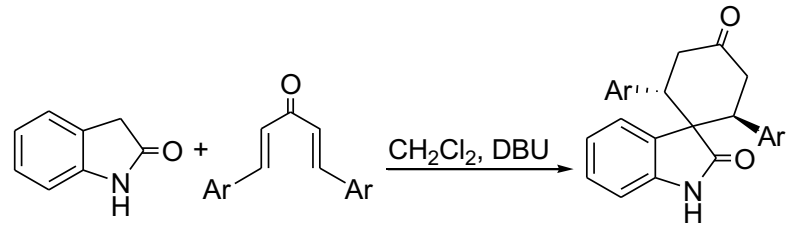

Synthesis of spiro[cyclohexanone-oxindoles].

Wang and co-worker have developed a methodology for the construction of spiro[cyclohexanone-oxindoles] through cascade [5+1] Michael/Michael addition reactions between divinyl ketones and $\mathrm{N}$-unprotected oxindoles catalyzed by combinations of cinchona-based chiral primary amines and $\alpha$-amino acid derivatives. The final products were obtained with good diastereoselectivities and enantioselectivities (Wu et al, 2012). 
<smiles>O=C(/C=C/c1ccccc1)/C=C/c1ccccc1</smiles>

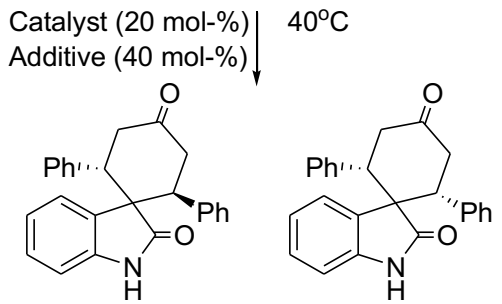

The reaction between divinyl ketones and $\mathrm{N}$-unprotected oxindoles catalyzed by combinations of cinchona-based chiral primary amines and $\alpha$-amino acid derivatives..

It had been witnessed in the last decade a growth of RCM (Rearranged Claisen-Michael) reaction as one of the powerful synthetic tools in organic synthesis (Grubbs and O'Leary, 2015). Its use in the synthesis of Spiro systems, however,was underexploration. It was found that there are very few reports on the synthesis of Spiro cyclic compounds based on RCM reaction. It was conceived that a combination of Claisen rearrangement and RCM reaction-based methodology developed (Srikrishna and Vasantha Lakshmi, 2005).

In the present work a new class of Spiro compounds, bisspirocyclohexanone with different substituted aryl group, are prepared by Michael reaction of diarylidene acetones with active methylene compound (Anthrone). The products of these reactions are of interest in terms of their stereochemistry and as starting materials for the synthesis of compounds with expected biological activity.

\section{EXPERIMENTAL}

\subsection{Materials}

Melting points were measured on Gallenkamp and uncorrected. UV-spectra were displayed using UV-visible spectrophotometer (1650-PC). IR spectra were recorded on FT-IR-600 Fourier-
Transform infrared spectrophotometer. ${ }^{1} \mathrm{H}-\mathrm{NMR}$ spectra were recorded by Bruker spectrometer $(400 \mathrm{MHz})$, using TMS as an internal standard and $\mathrm{CDCl}_{3} / \mathrm{D}_{6}$-DMSO as solvents. ${ }^{13} \mathrm{C}-\mathrm{NMR}$ spectra were taken on Bruker spectrometer (75.5 MHz).

Condensation of acetone with benzaldehyde and substituted benzaldehydes

The steps of the original procedure had been followed to get arylidene acetones compared with the authentic samples showing a good agreement of their physical properties and spectral data. m.p. $=38-40{ }^{\circ} \mathrm{C}$, UV $\left(\mathrm{CHCl}_{3} \lambda \max .=292 \mathrm{~nm}\right), \mathrm{IR}\left(\mathrm{KBr}, v \mathrm{~cm}^{-1}\right)$ : $1666(\mathrm{C}=\mathrm{O}), 1612(\mathrm{C}=\mathrm{C}), 1450(\mathrm{C}=\mathrm{C}$.<smiles>[X]c1ccc(/C=C/C(C)=O)cc1</smiles>

Structure of Arylidene acetone.

Condensation of arylidene acetones with different benzaldehydes (Al-Hamdany et al, 2014).

To a cold stirred mixture of the substituted benzaldehydes $(0.03 \mathrm{~mole})$ and arylidene acetone $(0.03 \mathrm{~mole})$ in $50 \mathrm{ml}$ absolute ethanol, $1 \mathrm{gm}$ of potassium hydroxide was added in small portion to the mixture in a period of $15 \mathrm{~min}$. The Stirring was continued for additional $1 \mathrm{~h}$. at room temperature. The resulting precipitate was then filtered off, washed with a little amount of cold ethanol and recrystallized from ethanol to get a solid product(1,3dibenzylidene acetone). Some physical properties and spectral data were illustrated in Tables (I, III, IV and V).

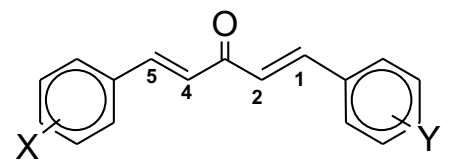

Structure of 1,3-Diarylidene acetone.

Table 1. Some physical properties of 1,3-diarylidene acetones

\begin{tabular}{|c|c|c|c|c|c|c|}
\hline $\begin{array}{l}\text { Comp } \\
\text {. No. }\end{array}$ & $\mathbf{X}$ & $\mathbf{Y}$ & Name of compound & Color & $\begin{array}{l}\text { m.p. } \\
\left({ }^{\circ} \mathrm{C}\right)\end{array}$ & $\begin{array}{c}\text { Yiel } \\
\text { d } \\
(\%)\end{array}$ \\
\hline 1 & $\mathrm{H}$ & $\mathrm{H}$ & $\begin{array}{l}\text { 1,5-diphenylpenta- } \\
\text { 1,4-dien-3-one }\end{array}$ & Yellow & $\begin{array}{l}99- \\
100\end{array}$ & 50 \\
\hline 2 & $\begin{array}{l}2- \\
\mathrm{Cl}\end{array}$ & $4-\mathrm{Br}$ & $\begin{array}{l}\text { 1-(4-bromophenyl)- } \\
\text { 5-(2-chlorophenyl) } \\
\text { penta-1,4-dien-3-one }\end{array}$ & $\begin{array}{c}\text { Pale } \\
\text { Yellow }\end{array}$ & $\begin{array}{c}174- \\
175\end{array}$ & 25 \\
\hline 3 & $\begin{array}{l}4- \\
\mathrm{Br}\end{array}$ & $\begin{array}{c}4- \\
\mathrm{MeO}\end{array}$ & $\begin{array}{l}\text { 1-(4- } \\
\text { methoxyphenyl)-5- } \\
\text { (4-bromophenyl) } \\
\text { penta-1,4- dien-3-one }\end{array}$ & Yellow & $\begin{array}{c}178- \\
180\end{array}$ & 50 \\
\hline 4 & $\mathrm{H}$ & $\begin{array}{c}3- \\
\mathrm{NO}_{2}\end{array}$ & $\begin{array}{l}\text { 1-(3-nitrophenyl)-5- } \\
\text { phenylpenta-1,4- } \\
\text { dien-3-one }\end{array}$ & $\begin{array}{c}\text { Pale } \\
\text { Yellow }\end{array}$ & $\begin{array}{c}130- \\
132\end{array}$ & 90 \\
\hline 5 & $\begin{array}{l}4- \\
\mathrm{Br}\end{array}$ & $4-\mathrm{Br}$ & $\begin{array}{l}\text { 1,5-bis(4- } \\
\text { bromopheny 1)penta- } \\
\text { 1,4-dien-3-one }\end{array}$ & $\begin{array}{c}\text { Pale } \\
\text { Yellow }\end{array}$ & $\begin{array}{c}180- \\
181\end{array}$ & 50 \\
\hline
\end{tabular}

Condensation of Anthrone with DAA (Hussein, 2016). A mixture of DAA (0.01 mole) and anthrone (0.01 mole) was magnetically stirred in the presence of $3 \mathrm{ml}$ of $50 \% \mathrm{NaOH}$ in absolute ethanol. The stirring was continued for $2 \mathrm{hrs}$. at $50{ }^{\circ} \mathrm{C}$ in
$10 \mathrm{ml}$ DMSO. Some physical properties and spectral data were illustrated in Tables (II, VI, VII, and VIII). 
<smiles>O=C1CC(Br)C2(c3ccccc31)c1ccccc1C(=O)CC2Br</smiles>

Fig. 7. Structure of final product (substituted Bis-spirocyclohexanones).

Table 2. Some physical properties of spirocyclohexanones

\begin{tabular}{|c|c|c|l|c|c|c|}
\hline $\begin{array}{c}\text { Comp } \\
\text { No. }\end{array}$ & $\mathbf{X}$ & $\mathbf{Y}$ & $\mathbf{\text { Name of compound }}$ & Color & $\begin{array}{c}\mathbf{m} . \mathbf{p .} \\
\left({ }^{\circ} \mathbf{C}\right)\end{array}$ & $\begin{array}{c}\text { Yield } \\
\mathbf{( \% )}\end{array}$ \\
\hline 6 & $\mathrm{H}$ & $\mathrm{H}$ & $\begin{array}{l}\text { 3,5-Diarylcyclohexan } \\
\text { one Spiro [4,9'] } \\
\text { anthrone }\end{array}$ & $\begin{array}{c}\text { Pale } \\
\text { paige }\end{array}$ & $\begin{array}{c}143- \\
146\end{array}$ & 60 \\
\hline 7 & $2-\mathrm{Cl}$ & $4-\mathrm{Br}$ & $\begin{array}{l}\text { 3-(4-Bromophenyl)-5- } \\
\text { (2-chlorophenyl } \\
\text { cyclohexanone[4]Spir } \\
\text { o[9] anthrone }\end{array}$ & $\begin{array}{c}\text { Pale } \\
\text { orange }\end{array}$ & $\begin{array}{c}133- \\
134\end{array}$ & 40 \\
\hline 8 & $4-\mathrm{Br}$ & $\begin{array}{c}4- \\
\mathrm{MeO}\end{array}$ & $\begin{array}{l}\text { 3-(4-bromophenyl)- } \\
\text { 5(4-methoxyphenyl } \\
\text { cyclohexanone[4]Spir } \\
\text { o[9] anthrone }\end{array}$ & $\begin{array}{c}\text { Dark } \\
\text { paige }\end{array}$ & $\begin{array}{c}166- \\
167\end{array}$ & 11 \\
\hline 9 & $\mathrm{H}$ & $\begin{array}{c}3- \\
\mathrm{NO} 2\end{array}$ & $\begin{array}{l}\text { 3-( phenyl)-5- (3-nitro } \\
\text { phenyl)cyclohexanone } \\
{[4] \text { Spiro[9] anthrone }}\end{array}$ & Brown & $\begin{array}{c}170- \\
172\end{array}$ & 75 \\
\hline 10 & $4-\mathrm{Br}$ & $4-\mathrm{Br}$ & $\begin{array}{l}\text { 3,5-Di(4-Bromophen } \\
\text { yl)-cyclohexanone } \\
\text { Spiro [4,9'] anthrone }\end{array}$ & Paige & $\begin{array}{c}137- \\
139\end{array}$ & 14 \\
\hline
\end{tabular}

\section{RESULTS AND DISSCUTION}

The acidic protons of acetone had been used to get a condensation product by the addition of one mole of benzaldehyde or substituted benzaldehydes to afford arylidene acetone as shown below (Fig. 8):

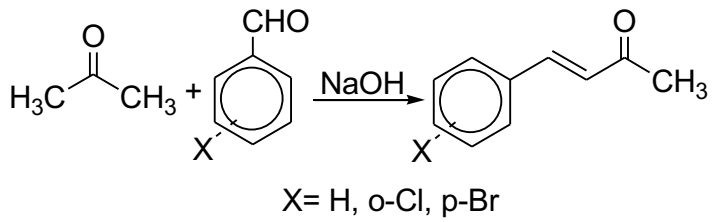

The Condensation of Aceton with Benzaldehyde.

The condensation of arylidene acetones with another mole of the same or different substituted benzaldehydes gave diarylidene acetones DAA(1-5) as shown on (Fig. 9):

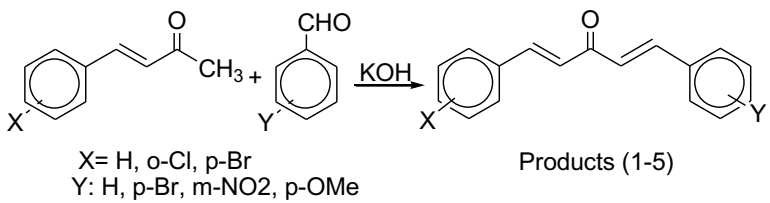

The Condensation of Arylidene Acetones with Benzaldehydes.

The spectral data (FTIR, U.V., ${ }^{1} \mathrm{H}-\mathrm{NMR}$ and ${ }^{13} \mathrm{C}-\mathrm{NMR}$ ) of DAA compounds, which prepared from the condensation between Arylidene Acetones with Benzaldehydes, were consistent with the structures of (1-5), Tables (III, IV and V).
Table 3. spectral data (ftir, uv) of 1,3-diarylidene acetones

\begin{tabular}{|c|c|c|c|c|}
\hline \multirow{2}{*}{ Comp. No. } & \multirow{2}{*}{$\begin{array}{c}\text { U.V. } \mathbf{C H C l}_{3} \\
\mathbf{n m}\end{array}$} & \multicolumn{3}{|c|}{ FTIR (KBr) $\mathbf{~ c m}^{-\mathbf{1}}$} \\
\cline { 3 - 5 } & 320 & 1651 & 1446 & 1626 \\
\hline 1 & 340 & 1653 & 1490 & 1618 \\
\hline 2 & 336 & 1650 & 1510 & 1593 \\
\hline 3 & 318 & 1668 & 1589 & 1624 \\
\hline 4 & 334 & 1649 & 1487 & 1624 \\
\hline 5
\end{tabular}

Table 4. spectral data $\left({ }^{13} \mathrm{C}-\mathrm{NMR}\right)$ of 1,3-diarylidene acetones

\begin{tabular}{|c|c|c|c|c|}
\hline \multirow{2}{*}{$\begin{array}{c}\text { Comp. } \\
\text { No. }\end{array}$} & \multicolumn{4}{|c|}{${ }^{\mathbf{1 3}} \mathbf{C}-\mathbf{N M R} \boldsymbol{\delta} \mathbf{p p m}$} \\
\hline 1 & $\boldsymbol{C}-\mathbf{2}, \boldsymbol{C}-\mathbf{4}$ & $\boldsymbol{C}-\mathbf{1}, \boldsymbol{C}-\mathbf{5}$ & $\boldsymbol{C}=\boldsymbol{O}$ & $\boldsymbol{A r} \boldsymbol{C}$ \\
\hline 2 & 131 & 152 & 188 & $127-129$ \\
\hline 3 & 130 & 151 & 188 & $127-132$ \\
\hline 4 & 130 & 152 & 188 & $115-132$ \\
\hline 5 & 132 & 153 & 188 & $126-130$ \\
\hline
\end{tabular}

Table 5. spectral data (1h-NMR) of 1,3-diarylidene acetones

\begin{tabular}{|c|c|c|c|c|c|}
\hline \multirow{2}{*}{$\begin{array}{c}\text { Comp. } \\
\text { No. }\end{array}$} & \multicolumn{5}{|c|}{${ }^{\mathbf{H}}$ H-NMR $\boldsymbol{\delta} \mathbf{p p m}$} \\
\cline { 2 - 7 } 1 & $\boldsymbol{H}-\mathbf{2}$ & $\boldsymbol{H}-4$ & $\boldsymbol{H}-\mathbf{1}$ & $\boldsymbol{H}-\mathbf{5}$ & $\boldsymbol{A}$ - $\boldsymbol{H}$ \\
\hline \multirow{2}{*}{1} & $6.7 \mathrm{~d}$ & $7.1 \mathrm{~d}$ & $7.99 \mathrm{~d}$ & $7.7 \mathrm{~d}$ & $7.2-7.4 \mathrm{~m}$ \\
& $(1 \mathrm{H})$ & $(1 \mathrm{H})$ & $(1 \mathrm{H})$ & $(1 \mathrm{H})$ & $(10 \mathrm{H})$ \\
\hline \multirow{2}{*}{2} & $6.7 \mathrm{~d}$ & $7.1 \mathrm{~d}$ & $8.1 \mathrm{~d}$ & $7.8 \mathrm{~d}$ & $7.2-7.4 \mathrm{~m}$ \\
& $(1 \mathrm{H})$ & $(1 \mathrm{H})$ & $(1 \mathrm{H})$ & $(1 \mathrm{H})$ & $(8 \mathrm{H})$ \\
\hline \multirow{2}{*}{3} & \multicolumn{2}{|c|}{$7.1 \mathrm{~d}$} & \multicolumn{2}{|c|}{$7.7 \mathrm{~d}$} & $6.8-7.5 \mathrm{~m}$ \\
& \multicolumn{2}{|c|}{$(2 \mathrm{H})$} & \multicolumn{2}{c|}{$(2 \mathrm{H})$} & $(8 \mathrm{H})$ \\
\hline \multirow{2}{*}{4} & $7.1 \mathrm{~d}$ & $7.4 \mathrm{~d}$ & $7.7 \mathrm{~d}$ & $7.8 \mathrm{~d}$ & $7.2-8.3 \mathrm{~m}$ \\
& $(1 \mathrm{H})$ & $(1 \mathrm{H})$ & $(1 \mathrm{H})$ & $(1 \mathrm{H})$ & $(8 \mathrm{H})$ \\
\hline
\end{tabular}




\begin{tabular}{|c|c|c|c|c|c|}
\hline \multirow{2}{*}{$\begin{array}{c}\text { Comp. } \\
\text { No. }\end{array}$} & \multicolumn{5}{|c|}{${ }^{1} \mathrm{H}-\mathrm{NMR} \delta \mathrm{ppm}$} \\
\hline & $H-2$ & $H-4$ & $H-1$ & H-5 & $A r-H$ \\
\hline 5 & \multicolumn{2}{|c|}{$\begin{array}{l}7.1 \mathrm{~d} \\
(2 \mathrm{H})\end{array}$} & \multicolumn{2}{|c|}{$\begin{array}{l}7.7 \mathrm{~d} \\
(2 \mathrm{H})\end{array}$} & $\begin{array}{c}7.3-7.4 \mathrm{~m} \\
(8 \mathrm{H})\end{array}$ \\
\hline
\end{tabular}

The prepared DAA's (1-5) had been condensed with anthrone in a strong basic medium to afford the title compounds (6-10) as shown in Fig. $10 . \quad$ below:<smiles>[X]c1ccc(/C=C/C(=O)/C=C/c2ccc([Y])cc2)cc1</smiles>

Products $(6-10)$

Michael Condensation of Anthron with DAA.

The FT-IR spectrum of products (spiro cyclohexanone 6-10) manifests a strong absorption band at $\left(1715 \mathrm{~cm}^{-1}\right)$ corresponds to stretching vibration of carbonyl group compared with corresponding 1,3-diarylidene acetones compounds $\left(1653 \mathrm{~cm}^{-1}\right)$ (Table III). This difference may be attributed to the absence of the conjugation and cyclization. Another absorption band appeared at $\left(1606 \mathrm{~cm}^{-1}\right)$ related to the stretching vibration of aromatic ring. The U.V spectra showed wavelengths at maximum absorption ( $\lambda$ max) 332-386 nm (Table VI).

The ${ }^{1} \mathrm{H}-\mathrm{NMR}$ for compound ( 6 ) as a representative model for the series showed a doublet signal for $4 \mathrm{H}$ resonates at $\delta 2.7 \mathrm{ppm}$ for $\mathrm{H}-2 \& \mathrm{H}-6$, while a triplet signal displayed at $\delta 3.6$ attributed to $2 \mathrm{H}$ of $\mathrm{H}-3$ and $\mathrm{H}-5$. The aromatic $18 \mathrm{H}$ seemed as a multiplet signal at a range of $\delta 7.13-7.87 .{ }^{13} \mathrm{C}-\mathrm{NMR}$ showed a signal at $\delta 45$ for $\mathrm{C}-2$ and $\mathrm{C}-6, \delta 40$ for $\mathrm{C}-3$ and $\mathrm{C}-5, \delta 56$ for $\mathrm{C}-4$. but a signal at $\delta$ 190 related to $\mathrm{C}=\mathrm{O}$ of anthrone, finally $\delta 212$ belongs to $\mathrm{C}=\mathrm{O}$ of $\mathrm{C}-1$, Tables (VII and VIII).

Table 5. spectral data (ftir, uv) of spirocyclohexanones

\begin{tabular}{|c|c|c|c|}
\hline \multirow{2}{*}{$\begin{array}{c}\text { Comp. } \\
\text { No. }\end{array}$} & \multirow{2}{*}{$\begin{array}{c}\text { U.V. } \mathbf{C H C l}_{\mathbf{n}} \\
\mathbf{n m}\end{array}$} & \multicolumn{2}{|c|}{ FTIR $(\mathbf{K B r}) \mathbf{c m}^{-\mathbf{1}}$} \\
\hline 6 & 332 & 1639 & $\boldsymbol{C}$ \\
\hline 7 & 386 & 1715 & 1601 \\
\hline 8 & 340 & 1637,1710 & 1606 \\
\hline 9 & 344 & 1637 & 1616 \\
\hline 10 & 382 & 1637 & 1616 \\
\hline
\end{tabular}

Table 6. spectral data $\left({ }^{13} \mathrm{C}-\mathrm{NMR}\right)$ of spirocyclohexanones

\begin{tabular}{|c|c|c|c|c|c|}
\hline \multirow{2}{*}{$\begin{array}{c}\text { Comp. } \\
\text { No. }\end{array}$} & \multicolumn{5}{|c|}{${ }^{13} \mathrm{C}-\mathrm{NMR} \delta \mathrm{ppm}$} \\
\hline & $\begin{array}{l}C-2, \\
C-6\end{array}$ & $\begin{array}{l}C-3, \\
C-5\end{array}$ & $C-4$ & $\begin{array}{c}C=O \text { of } \\
\text { Anthrone }\end{array}$ & $\begin{array}{c}C=O \text { of } \\
\text { Cyclohexanone }\end{array}$ \\
\hline 6 & 45 & 40 & 56 & 190 & 212 \\
\hline 7 & $\begin{array}{l}44 \\
46\end{array}$ & $\begin{array}{l}41 \\
36\end{array}$ & 58 & 191 & 213 \\
\hline 8 & 44 & 40 & 54 & 188 & 210 \\
\hline 9 & 44 & 39 & 56 & 192 & 211 \\
\hline 10 & 45 & 41 & 57 & 190 & 212 \\
\hline
\end{tabular}

Table 7. spectral data $\left({ }^{1} \mathrm{~h}-\mathrm{NMR}\right)$ of spirocyclohexanones

\begin{tabular}{|c|c|c|c|}
\hline \multirow{2}{*}{$\begin{array}{c}\text { Comp. } \\
\text { No. }\end{array}$} & \multicolumn{3}{|c|}{${ }^{\mathbf{1}} \mathbf{H}-\mathbf{N M R} \boldsymbol{\delta} \mathbf{~ p p m}$} \\
\hline \multirow{2}{*}{6} & $\boldsymbol{H - 2 , \boldsymbol { H } - \mathbf { 6 }}$ & $\boldsymbol{H}-\mathbf{3} \boldsymbol{H}-\mathbf{5}$ & $\boldsymbol{A r}-\boldsymbol{H}$ \\
\hline \multirow{2}{*}{7} & $2.7 \mathrm{~d}$ & $3.6 \mathrm{t}$ & $7.1-7.8 \mathrm{~m}$ \\
& $(4 \mathrm{H})$ & $(2 \mathrm{H})$ & $(18 \mathrm{H})$ \\
\hline \multirow{2}{*}{8} & $2.6 \mathrm{~d}$ & $3.5 \mathrm{t}$ & $7.1-7.9 \mathrm{~m}$ \\
& $(4 \mathrm{H})$ & $(2 \mathrm{H})$ & $(16 \mathrm{H})$ \\
\hline & $2.5 \mathrm{~d}$ & $3.6 \mathrm{t}$ & $6.8-7.9 \mathrm{~m}$ \\
$(4 \mathrm{H})$ & $(2 \mathrm{H})$ & $(16 \mathrm{H})$ \\
\hline
\end{tabular}

\begin{tabular}{|c|c|c|c|}
\hline \multirow{2}{*}{$\begin{array}{c}\text { Comp. } \\
\text { No. }\end{array}$} & \multicolumn{3}{|c|}{${ }^{\mathbf{H}} \mathbf{H}-\mathbf{N M R} \boldsymbol{\delta} \mathbf{p p m}$} \\
\cline { 2 - 4 } & $\boldsymbol{H - 2 , \boldsymbol { H } - \mathbf { 6 }}$ & $\boldsymbol{H}-\mathbf{3}, \boldsymbol{H}-\mathbf{5}$ & $\boldsymbol{A}$ - $\boldsymbol{H}$ \\
\hline \multirow{2}{*}{10} & $2.6 \mathrm{~d}$ & $3.4 \mathrm{t}$ & $7.2-7.9 \mathrm{~m}$ \\
& $(4 \mathrm{H})$ & $(2 \mathrm{H})$ & $(17 \mathrm{H})$ \\
\hline \multirow{2}{*}{10} & $2.7 \mathrm{~d}$ & $3.7 \mathrm{t}$ & $7.1-7.9 \mathrm{~m}$ \\
& $(4 \mathrm{H})$ & $(2 \mathrm{H})$ & $(16 \mathrm{H})$ \\
\hline
\end{tabular}

The suggested mechanism for the reaction of (DAA) and anthrone described in fig. 13. It may follow the formation of the anion, fig. 11 , which may in turn attack the $\alpha, \beta$ unsaturated system of the (DAA) through two stages:<smiles>O=C1c2ccccc2[C-]c2ccccc21</smiles>

Structure of the first anion.

The anion may attack the $\beta$ - carbon via Michael addition to afford M1, which may lose another acidic proton under the strong basic conditions to afford the second anion, fig. 12, which in turn may attack the $\beta^{\prime}-$ carbon via intramolecular Michael addition to produce the expected final spiro product.

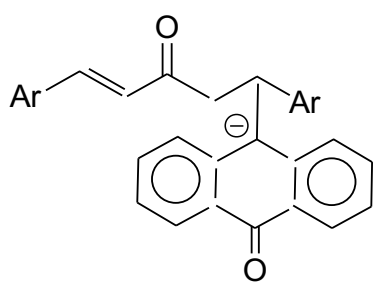

Structure of the second anion.<smiles>[X]c1ccc(/C=C/C(=O)/C=C/c2ccc([Y])c(O[Na])c2)cc1</smiles><smiles>[X]c1ccc(/C=C/C(=O)CC(c2cccc([X])c2)C2(c3ccccc3)c3ccccc3C(=O)c3ccccc32)cc1</smiles>

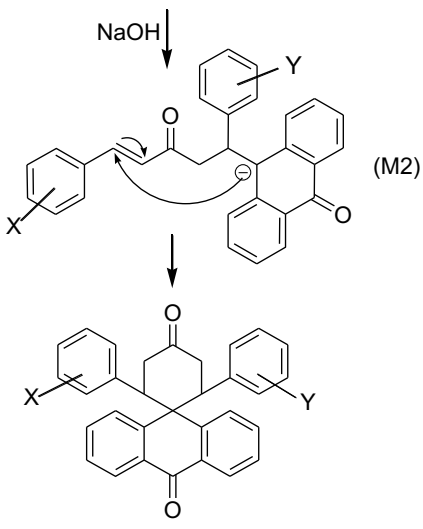

Final spiro product $(6-10)$

The suggested mechanism for the reaction of (DAA) and anthrone to produce the expected final spiro product. 


\section{Conclusion}

The two acidic protons of anthrone were the basis of the reaction, i.e. under strong basic conditions these protons will be abstracted to afford the corresponding carbanions which act as nucleophiles added as intermolecular Michael addition to DAA's to give the Spiro products.

\section{REFERENCES}

A. A. Raj, and R. Raghnathan, "Synthesis of spiropyrrolidine via formal $[3,2]$ cycloaddition of unusual enones and cis 3benzoy 1-1-cyclohexy 1-2-phenylaziridine," Tetrahedron, vol. 59, pp. 2907-2911, 2003.

A. Dandia, R. Singh, S. Khaturia, C. Merienne, G. Morgant, and A. Loupy, "Efficient microwave enhanced regioselective synthesis of a series of benzimidazolyl/triazolyl spiro[indolethiazolidinones] as potent antifungal agents and crystal structure of spiro[3H-indole-3,2'- thiazolidine]-3'(1,2,4triazol-3-yl)-2,4'(1H)-dione," Bioorg. Med. Chem., vol. 14, pp. 2409-2417, 2006.

A. J. Al-Hamdany, M. S. Al-Jawady, R. A. Saeed, "Synthesis and Spectral Charactrization of Some Pyrimidinones," Raf. J. Sci., vol. 25(3), pp. 16-23, 2014.

A. Scala, M. Cordaro, G. Grassi, A. Piperno, and G. Barberi, "Direct synthesis of C3-mono-functionalized oxindoles from $\mathrm{N}$ unprotected 2-oxindole and their antileishmanial activity," Bioorg. \& Med. Chem., vol. 22, pp.1063-1069,2014.

A. Srikrishna and B. Vasantha Lakshmi, "Construction of vicinal quaternary carbon atoms by Ireland ester Claisen rearrangement: total synthesis of $( \pm)$-herbertenolide, $( \pm)$ herberteneacetal, $( \pm)$-herbertene-1,14-diol and $( \pm)$-herbertene1,15-diol," Tetrahedron Lette.,vol. 46, pp. 4879, 2005.

B. Wu, J. Chen, M. Li, J. Zhang, and X. Wang, "Highly Enantioselective Synthesis of Spiro[cyclohexanone-oxindoles] and Spiro[cyclohexanone-pyrazolones] by Asymmetric Cascade
[5+1] Double Michael Reactions," Eur. J. Org. Chem., pp.1318-1327, 2012.

Ch. Marti and E.Carreira, "Total Synthesis of (-)-Spirotryprostatin B: Synthesis and Related Studies," J.Am. Chem. Soc, vol. 127, pp. 11505-11515, 2005.

I. Ungureanu, Ph. Klotz, A. Schoenfelder and A. Mann," 2-Phenyl-Ntosylazetidine as a formal 1,4 dipole precursor," Chem. Commun., pp. 958-959, 2001.

J. Jayashankaran, R. D. R.S Manian, and R. Raghunathan, “A facile entry into a novel class of dispiroheterocycles through 1,3dipolar cycloaddition," ARKIVOC., vol. 11, pp. 32-39, 2005.

J. Ma, and S.M. Hecht, "Javaniside, a novel DNA cleavage agent from Alangium javanicum having a unusual oxindole skeleton," Chem. Commun., pp. 1190-1191, 2004.

K. Aggarwal, and K. Vij, "An efficient catalyst free synthesis of nitrogen containing spiro heterocycles via $[5+1]$ double Michael addition reaction," RSC Adv., vol. 4, pp. 13313, 2014.

K. Ding, Y. Lu, N.Z. Coleska, S. Qiu, and Y. Ding, "Structure-Based Design of Potent Non-Peptide MDMZ Inhibitors" J. Am. Chem. Soc., vol. 127, pp. 10130-10131, 2005.

L. K. Smith, and I. R. Baxendale, "Total syntheses of natural products containing spirocarbocycles," Org. Biomol. Chem., vol. 13, pp. $9907,2015$.

M. G. Assy, E. K. Mohamed, and A. S. Mohamed, "Heterocyclization of barbituric acid: Synthesis of novel condensed pyrimidines Reda A. Haggam,” Inter. J. of Adv. Res., vol. 3, pp. 692-698, 2015.

M. Ghandi, A. Yari, S. Jamal, and A. Taheri, "Synthesis of novel spiropyrrolidine through 1,3-dipolar cycloaddition," Tetrahedron Lett., vol. 50, pp. 4724-472, 2009.

M. S. Hussein, "Synthesis, Characterization and Antibacterial Evaluation of Some Substituted Pyrrolidines," Chem. Sci. Intern. J. , vol 17(2), pp. 1-8, 2016. 